\title{
Diffusion institutionnelle de la médecine chinoise
}

Typologie des principaux enjeux

Pierre-Henry De Bruyn et Évelyne Micollier

\section{(2) OpenEdition \\ Journals}

Édition électronique

URL : https://journals.openedition.org/chinaperspectives/5624

DOI : $10.4000 /$ chinaperspectives.5624

ISSN : 1996-4617

Éditeur

Centre d'étude français sur la Chine contemporaine

Édition imprimée

Date de publication : 1 octobre 2011

Pagination : 24-33

ISSN : 2070-3449

Référence électronique

Pierre-Henry De Bruyn et Évelyne Micollier, « Diffusion institutionnelle de la médecine chinoise », China Perspectives [En ligne], 2011/3 | 2011, mis en ligne le 30 octobre 2011, consulté le 16 février 2022.

URL : http://journals.openedition.org/chinaperspectives/5624; DOI : https://doi.org/10.4000/

chinaperspectives.5624 


\title{
Diffusion institutionnelle de la
}

\section{médecine chinoise}

\author{
Typologie des principaux enjeux
}

\author{
PIERRE-HENRY DE BRUYN ET ÉVELYNE MICOLLIER*
}

\begin{abstract}
RÉSUMÉ : Le présent article vise à proposer une typologie des différents enjeux que rencontre aujourd'hui la diffusion de la médecine traditionnelle chinoise dans le monde en mettant successivement en lumière des difficultés idéologiques, épistémologiques, politiques, économiques et didactiques. Après avoir montré l'aspect polarisé des débats universitaires concernant la médecine chinoise, est abordée la question du statut épistémologique de cette tradition chinoise en la confrontant à la biomédecine dominante d'origine occidentale. L'originalité des structures politiques chinoises mises en place pour protéger et promouvoir cette tradition nationale est ensuite mise en valeur comme source possible d'inspiration au niveau international avant que soient décrits les différents facteurs économiques susceptibles de jouer positivement ou négativement sur l'évolution de ce patrimoine médical et culturel au niveau local. Les questions didactiques spécifiques que pose la transmission de ce patrimoine et l'enseignement de cette discipline sont finalement analysées avant d'en tirer conclusion.
\end{abstract}

MOTS CLÉS : Médecine chinoise, épistémologie, politique de santé, pédagogie, histoire des sciences, anthropologie des sciences

\section{$\mathrm{L}$} 'enseignement de la médecine chinoise à un public occidental presque totalement ignorant des principes philosophiques de la civilisation chinoise implique de surmonter un ensemble de difficultés intellectuelles, pédagogiques et institutionnelles considérable ${ }^{(1)}$. En 2004, le professeur Éric Marié, qui avait à cette époque un poste de directeur de recherche à la faculté de médecine chinoise de Nanchang au Jiangxi et enseignait également cette discipline à l'université de Lausanne (2), avait été invité à apporter sa compétence pour tenter de mettre en place, en France, à l'université de La Rochelle, un diplôme universitaire susceptible de contribuer à répondre à ce défi. Ce diplôme, d'abord soutenu par une lettre du conseiller d'éducation à l'ambassade de Chine en France au président de l'université, puis approuvé par le conseil d'administration, reçut de nombreuses demandes $d^{\prime}$ inscription mais ne vit pourtant jamais le jour. Les formulaires d'inscription furent renvoyés aux étudiants. Aucune explication ne fut jamais avancée sur les raisons justifiant que cette formation ne soit finalement pas ouverte. Cette expérience d'échec conduisit Pierre-Henry de Bruyn, à l'initiative de ce projet, à réfléchir aux résistances que la diffusion de la tradition médicale chinoise rencontre aujourd'hui dans un certain nombre de pays occidentaux pour pénétrer dans le milieu universitaire. Le présent article est le fruit de cette réflexion, enrichie par celle qu'Évelyne Micollier mène sur le système de santé chinois depuis une vingtaine d'années. Le travail ci-dessous consistera à tracer une typologie des enjeux d'ordre idéologique, épistémologique, politique, économique et didactique qui se cristallisent autour de la médecine chinoise dans son processus de diffusion pour tenter d'en dégager quelques considérations quant à son avenir. Dans la ligne de ce dossier spécial, nous considèrerons ici la médecine chinoise comme un patrimoine du monde entier et non pas comme un phénomène purement chinois ou local. Cela nous permettra d'illustrer notre réflexion à partir d'observations faites tantôt en Chine et en Asie orientale, tantôt en Occident.

\section{Premier type d'enjeu : éviter une bipolarisation idéologique caricaturale}

Le premier genre de difficultés que l'on rencontre en parlant de médecine chinoise en milieu universitaire provient du caractère rapidement idéologique que prennent les discussions sur ce sujet. Depuis quelques années, ce problème existe aussi en Chine comme on a pu l'observer en 2006 avec le débat très médiatisé autour d'un article de Zhang Gongyao, professeur de

Pierre-Henry de Bruyn est chercheur au CEFC (UMIFRE n 18 / CNRS-USR nº 3331 Asie Orientale) et rédacteur en chef de Perspectives chinoises; Évelyne Micollier est chercheure à l'IRD (Institut de recherche pour le développement), UMI 233 (Unité mixte internationale), IRD-Université de Montpellier I. Elle a étudié la médecine chinoise à l'Institut de médecine chinoise de Canton au début des années 1990 dans le cadre de sa recherche doctorale « Un aspect de la pluralité médicale en Chine populaire : les pratiques de qigong, dimension thérapeutique et dimension sociale », thèse de doctorat en anthropologie, Université de Provence, 1995

1. Etant donné que le terme de « médecine traditionnelle chinoise » (MTC), choisi par la RPC à partir des années 1950 pour traduire le terme chinois zhongyi (« médecine chinoise »-MC) prend de plus en plus un sens restreint qui n'est plus vraiment synonyme de "médecine chinoise", nous préférerons employer ici une traduction littérale du terme même si nous utiliserons encore le sigle « MTC » par commodité. Il importe de rappeler à ce sujet qu'Elisabeth Hsu, Volker Scheid et Kim Taylor ont explicité la différence entre la médecine traditionnelle promue par le gouvernement, dénommée MTC, et la médecine chinoise dans son acceptation plus large. En présentant la pluralité des pratiques médicales, Scheid puis Taylor rendent précisément compte des évolutions de la MTC depuis 1949 : la médecine chinoise a été transformée en MTC par une intervention étatique planifiée ». (Évelyne Micollier, « Un savoir thérapeutique hybride et mobile - Éclairage sur la recherche médicale en médecine chinoise en Chine aujourd'hui », Revue d'anthropologie des connaissances, vol. 5, n 1, 2011, p. 41-70; p. 47). Cet extrait renvoie aux études suivantes : Elisabeth Hsu, The Transmission of Chinese Medicine, Cambridge UK, Cambridge University Press, 1999 ;--, Chinese Medicine: Innovation, Convention and Controversy, Cambridge UK, Cambridge University Press, 2001 ; Volker Scheid, Chinese Medicine in Contemporary China - Plurality and Synthesis, Durham - Londres, Duke University Press, 2002 ; et Kim Taylor, Chinese Medicine in Early Communist China, 1945 - 1963. A Medicine of Revolution, Londres, Routledge, 2005. De plus, on notera aussi (p. 48) que « dans les traductions officielles aujourd'hui, les auteurs chinois, comme le ministère de la Santé, confondent MTC avec Zhongyi ».

2. En 2003 , Éric Marié présenta également une thèse de doctorat en histoire comparée de la médecine à l'École des hautes études en sciences sociales. Ce travail fut ensuite publié sous le titre : Le diagnostic par les pouls en Chine et en Europe - Une histoire de la sphygmologie des origines au XVIII siècle, ParisBerlin-New York, Springer, 2011. 
philosophie des sciences de l'université de Changsha, qui remettait en cause l'insertion de la MTC dans le système de santé publique chinois ${ }^{(3)}$. Cependant, en Occident, cet aspect idéologique est d'autant plus marqué qu'il déborde aussi, comme nous le montrerons, le champ étroit des quelques spécialistes de cette discipline. Le caractère « national » de la médecine « chinoise » réveille inconsciemment dans le monde occidental ces réflexes profonds qui déterminent l'attitude des êtres humains face à l'étranger. Dans l'introduction d'un livre sur les pratiques d'acupuncture en Occident publié en 1997, Giovanni Maciocia faisait ainsi remarquer que :

\section{Depuis que la médecine orientale a été introduite dans le monde} occidental, du moins à l'époque moderne, il y a eu une tension dynamique entre d'une part le besoin d'absorber, de comprendre et de préserver la médecine orientale et, d'autre part, le besoin de l'adapter aux conditions occidentales. On pourrait présenter deux perspectives opposées, la première invoquant le besoin d'adapter doucement la médecine orientale aux conditions occidentales, la seconde évoquant le besoin d'absorber et de maîtriser d'abord la médecine orientale telle qu'elle est pratiquée en Asie ${ }^{(4)}$.

Les discours occidentaux sur la médecine chinoise sont donc traversés par une tension entre un mouvement centripète et un autre centrifuge. L'approche centripète consiste à tenter d'absorber l'altérité de l'autre en filtrant le contenu de son savoir et de son expérience par les catégories de pensée coutumières. On la retrouve par exemple souvent dans les facultés de médecine occidentale. Dans ce genre de milieu, les seuls aspects de la médecine chinoise jugés dignes d'intérêts sont ceux qui, de façon fractionnée, peuvent rejoindre le « centre » du médecin occidental, ou autrement dit sa façon de penser : la tradition médicale étrangère cesse d'être " chinoise » mais certains aspects sont considérés comme méritant peut-être un intérêt « médical » en raison de leur efficacité thérapeutique (5). À l'opposé, l'approche centrifuge cherchera à mettre en valeur le caractère exotique de la discipline : elle est cultivée par de nombreuses écoles privées de médecine chinoise mais aussi en milieu universitaire dans les facultés d'études chinoises où le caractère « chinois » l'emporte sur la dimension médicale : la médecine chinoise devient alors une chinoiserie parmi d'autres.

Il importe de souligner qu'aucun milieu, y compris celui des spécialistes de la médecine chinoise, n'échappe à cette tension. On observe par exemple que les historiens occidentaux de la médecine chinoise présentent souvent, en fonction de leurs préjugés intellectuels ou idéologiques, des divergences de point de vue quant à leurs interprétations de l'histoire contemporaine. Ainsi Elisabeth $\mathrm{Hsu}$, anthropologue, dans la préface de son ouvrage collectif Innovation in Chinese Medicine, introduit les articles de Volker Scheid et de Kim Taylor, spécialistes de l'histoire de la médecine chinoise, en soulignant le contraste suivant :

Lorsque Taylor insiste sur l'unification réalisée par un endoctrinement politique, Scheid insiste plutôt sur le pluralisme existant dans les institutions gouvernementales; là où Taylor mentionne une application des sciences soviétiques, Scheid souligne l'adaptation aux médecines occidentales; et quand Taylor montre comment la connaissance canonique avait été abandonnée, Scheid présente la situation actuelle en détaillant les lignages médicaux et en établissant les généalogies de prescriptions médicales contemporaines ${ }^{(6)}$.
Ce contraste entre des préjugés positifs à l'égard de la médecine chinoise comme ceux de Volker Scheid ou négatifs, comme chez Kim Taylor, n'est pas nouveau. Le parallèle entre deux autres spécialistes comme Manfred Porkert et Paul Unschuld le confirme : le premier défend le caractère profondément scientifique de la médecine chinoise ; le second considère que le recours à cette discipline en Occident répond principalement à un vide idéologique et spirituel.

Manfred Porkert estime en effet que:

Actuellement trois facteurs majeurs travaillent ensemble à une " déstructuration fondamentale » (debasement) de la médecine chinoise. Le premier, le plus visible et le plus effrayant, est la manifeste puissance de la médecine occidentale orthodoxe, qui semble invincible et universelle. Le second, très répandu et pour lequel beaucoup de publicité est faite dans tous les pays industrialisés, est le soutien et l'intérêt que la médecine chinoise attire de la part de praticiens d'une médecine dite « alternative ${ }^{(7)} 》$. Et enfin, la menace de loin la plus sérieuse est la déformation et la détérioration dont la médecine chinoise souffre par la cause même de certains de ses porte-paroles les plus éminents en Chine proprement dite ${ }^{(8)}$.

Et pourtant, toujours selon Porkert, la médecine chinoise serait « une science de plein droit » (a science in its own right) dont l'existence offrirait à la profession médicale une chance unique de développements potentiels, pour peu qu'elle soit reconnue à ce titre ${ }^{(9)}$. C'est dans ce même esprit que certains auteurs entreprennent aujourd'hui de rédiger de véritables tableaux chronologiques comparatifs, attribuant ainsi indirectement à l'histoire de la médecine chinoise une importance comparable à celle de la médecine occidentale. Un des exemples les plus éminents de ce genre de travaux est la collection inaugurée en 2003 sous le titre très parlant de Bridging Hippocrates and Huang $\mathrm{Ti}^{(10)}$.

À l'opposé d'une telle façon de voir, Paul Unschuld estime plutôt que c'est l'idolâtrie actuelle de la « nature » en Occident qui...

3. Voir Évelyne Micollier, « Un savoir thérapeutique hybride et mobile - Éclairage sur la recherche médicale en médecine chinoise en Chine aujourd'hui », Revue d'anthropologie des connaissances, vol. 5, n 1, 2011, p. 41-70, p. 49 ;--, " Gestion de l'épidémie et usages de la médecine chinoise. Traitements et recherche en médecine chinoise traditionnelle dans le contexte de l'extension du traitement médical et social du SIDA en Chine », Perspectives chinoises, n 106, 2009, p. 75-87, p. 81.

4. Giovanni Maciocia, « Foreword », in Hugh MacPherson, Ted J. Kaptchuk (éd.), Acupuncture in PracticeCase History Insights from the West, Londres, Elsevier Limited, 1997, p. ix.

5. L'article de Lucia Candelise dans ce dossier montre qu'en France, il y a eu, parmi les médecins acupuncteurs, une forte réaction contre cette tendance centripète qui a engendré parmi eux un courant qu'elle appelle « traditionnaliste ». Voir aussi Ronald Guilloux « Évolution de la "tradition" dans la réception de l'acupuncture chinoise en France (1860-1980) », Revue d'anthropologie des connaissances, vol. 5, $n^{\circ} 1,2011$, p. 13-40.

6. Elisabeth Hsu (éd.), Innovation in Chinese Medicine, Cambridge, Cambridge University Press, 2001, p. 8.

7. La médecine chinoise se trouverait ainsi réduite à n'être plus qu'une technique thérapeutique holistique un peu éthérée manipulée par des partisans du nouvel âge et des médecines naturelles.

8. Manfred Porkert, Chinese Medicine Debased - Essays on Methodology, Dinkelscherben (Allemagne), Phainon, 1998, p. 2. Dans ce troisième point, Manfred Porkert vise directement les institutions étatiques chinoises actuelles qui répandent un enseignement de médecine chinoise davantage réfléchi sous la pression de mobiles politiques que celui d'un authentique souci de fidélité scientifique à une tradition thérapeutique. Dans les années 2000, aux mobiles politiques décrits par Porkert, s'ajoutent des mobiles visant à accroître le rayonnement scientifique de la Chine ainsi que des mobiles économiques puissants (Évelyne Micollier, « Gestion de l'épidémie et usages de la médecine chinoise. Traitements et recherche en médecine chinoise traditionnelle dans le contexte de l'extension du traitement médical et social du SIDA en Chine », art. cit., p. 81-82).

9. Manfred Porkert, Chinese Medicine Debased - Essays on Methodology, op. cit., p. 13-35 ; p. 34

10. Paolo Marandola (éd. et al.), A Comparative Thesaurus of Traditional Chinese and Western Urology, Andrology and Sexology, Amsterdam, Elsevier, col. « Bridging Hippocrates and Huang Ti », vol. 1, 2003. 
... demandait une théologie, et que celle-ci n'était pas offerte par l'usage de l'eau, de la chaleur, de la lumière et de l'air. C'est trop primitif. La soi-disant médecine chinoise était mieux équipée pour fournir la théologie demandée. Elle se présentait en même temps ellemême comme une religion séculière. [...] La " théologie » de la médecine chinoise fournit des réponses dont les croyants des Églises de religions conventionnelles doivent se passer. Évidemment, cette « théologie » n'en est pas véritablement une, car elle n'a pas de theos (dieu). Le yin-yang et la doctrine des cinq agents sont la cosmologie d'une religion séculière. C'est une religion car elle rend l'intégration des individus dans le grand tout complètement compréhensible. Elle est « séculière » parce que le numineux n'existe pas dans cette religion. Pas de dieu ni d'esprits. Pas de démons ni d'ancêtres(11).

On retrouve ici, au sujet de la MTC, le vieux débat occidental entre science et religion. Déchirée entre un possible statut de « science » (défendu par ceux qui non seulement l'étudient mais l'utilisent comme praticiens, à l'exemple de Volker Scheid ou Manfred Porkert), et une sorte de doctrine religieuse dans laquelle il faudrait croire ou dont on devrait douter (comme le suggèrent, chacun à leur façon, Paul Unschuld ou Kim Taylor), la médecine chinoise peine à trouver ce statut épistémologique qui constitue, par conséquent, le second enjeu fondamental qu'elle rencontre dans son processus de diffusion.

\section{Deuxième type d'enjeu : sortir d'un statut épistémologique flou}

La question du rapport entre science et religion s'est posée de façon très différente en Chine et en Occident. Le savoir ne s'est pas construit en Chine en opposition à un pouvoir religieux. II ne s'est pas non plus structuré dans un discours à prétention d'universalité scientifique. En tant que tradition « médicale », la motivation principale pour laquelle les Chinois ont cultivé et transmis leur tradition médicale est essentiellement pratique : seule comptait l'efficacité thérapeutique qu'elle permettait.

Or, pour arriver à des résultats thérapeutiques concrets, ils ont dû se pencher sur des êtres humains malades et en bonne santé. Ils l'ont fait avec, inconsciemment, une anthropologie et une cosmologie culturellement situées. La médecine chinoise est donc « chinoise » parce qu'elle repose sur une approche du corps et de l'esprit nourrie par une civilisation précise : la culture chinoise. Dans un monde où la culture occidentale a exercé une influence proche du raz-de-marée culturel, la médecine chinoise offre la possibilité de découvrir concrètement comment une action thérapeutique est possible à partir d'une autre perception de l'être humain et du monde que celle qu'offre la perspective occidentale. Shigehisa Kuriyama a montré combien la différence entre les perceptions du corps entre les deux civilisations grecque et chinoise est radicale dans des domaines aussi fondamentaux que l'histoire de la perception tactile, des relations entre les façons d'entendre et de voir ou encore des saignées :

Nous supposons habituellement que la structure profonde et les fonctionnements du corps humain sont une réalité universelle semblable partout. Or, lorsque nous plongeons dans l'histoire, notre sens des réalités est ébranlé [...] en constatant que les visions du corps présentées par diverses traditions médicales apparaissent fréquemment être tellement étrangères les unes aux autres que l'on croit avoir affaire à des mondes sans relations mutuelles (12).
Le constat de cette différence entre les perceptions du corps véhiculées par le taoïsme et celles qui circulent en Occident est à la base d'un ouvrage récent de Pierre-Henry de Bruyn qui, en prolongement du livre fondateur sur le corps taoïste de Kristoffer Schipper ${ }^{(13)}$, visait à dresser l'état actuel de cette question dans les études occidentales ${ }^{(14)}$. Ce travail présentait le taoïsme comme une doctrine centrée essentiellement sur le corps. Cependant, insister outre mesure sur les racines taoïstes (voire confucianistes ou bouddhistes) de la médecine chinoise risquerait également d'obscurcir le statut épistémologique de cette dernière plutôt que d'aider à le définir. Il convient par conséquent plutôt de poser autrement le problème en revenant à l'essentiel qu'est l'acte de soigner : c'est ce que les Chinois ont fait durant des siècles en transmettant leurs traditions médicales.

Avant le XIXe siècle, la seule « médecine » (醫學 - yixue) que connaissaient les Chinois était chinoise. Au cours de sa très longue histoire, la médecine chinoise fut néanmoins traversée par d'autres traditions médicales asiatiques, dont par exemple les médecines indiennes et bouddhistes, comme l'a souligné Paul Unschuld (15). Par ailleurs, elle fut aussi influencée par de nombreuses traditions médicales très locales ainsi que par les connaissances médicales d'autres groupes ethniques qui se réfèrent à des savoirs médicaux s'appuyant sur des sources écrites (médecines tibétaine, mongole, coréenne et ouïghoure) ou orales. L'irruption de la médecine importée par les Occidentaux dite 《 médecine occidentale » (西醫 - xiyi) a bouleversé ce paysage intellectuel et la tradition médicale chinoise se trouva progressivement relativisée en Chine au point de devenir bientôt seulement une médecine « chinoise » (zhongyi). Très vite certains médecins chinois tentèrent de proposer une synthèse en cherchant comment intégrer des éléments de la médecine occidentale à leur système médical traditionnel : ils seront connus comme « l'école de l'intégration entre les médecines chinoise et occidentale » (中西會通牌 - zhongxi huitong pai) ${ }^{(16)}$. Des années plus tard Mao Zedong relancera ces efforts en prônant une union des médecines occidentale et chinoise. Les débats seront nombreux sur la sorte d'union à envisager et tourneront autour de différents slogans. Dans quel ordre fallait-il penser les priorités : « d'abord la médecine chinoise puis la médecine occidentale » (先中後西 - xian zhong hou xi) ou bien l'inverse (17) ? Devait-on penser en termes $d^{\prime}$ « intégration » (結合 jiehe) mutuelle, d'« unification » (團結 - tuanjie), de « coopération » (合 作 - hezuo $)^{(18)}$, ou même encore de « synthèse » (綜合 - zonghe) ${ }^{(19)}$ ?

Cependant, certains chercheurs ou acteurs sociaux estiment que c'est plutôt à partir de sa propre spécificité, et non pas en cherchant à s'unir

11. Paul Unschuld (trad. par Karen Reimers), What Is Medicine? Western and Eastern Approaches to Healing, Berkeley/Los Angeles, University of California press, 2009, p. 202.

12. Shigehisa Kuriyama, The Expressiveness of the Body - and the Divergence of Greek and Chinese Medicine, New York, Zone Books, 1999, p. 8.

13. Kristofer Schipper, Le corps taoïste, Paris, Fayard, 1982.

14. Pierre-Henry de Bruyn, Le taoïsme - Chemins de découvertes, Paris, CNRS, 2010.

15. Paul Unschuld, Medicine in China: A History of Ideas, Berkeley, University of California Press, 1985.

16. Tang Zonghai (1851-1908), Zhang Shouyi (1873-1934) et Zhang Xichun (1860-1933), furent, entre autres, de célèbres partisans de cette tendance.

17. Kim Taylor, Chinese Medicine in Early Communist China, 1945 - 1963. A Medicine of Revolution, op. cit., p. 125.

18. Ibid., p. 137

19. Dans les années 2000, se répand l'utilisation de termes se référant à la notion de synthèse dans des colloques, des publications officielles, académiques ou populaires; par exemple dans le cadre du 1er Congrès mondial de l'OMS sur la médecine traditionnelle (chuantong yixue) (WHO Congress on Traditional Medicine hosted by the Ministry of Health and SATCMC, 7-9 novembre, 2008, qui portait sur le thème « Développer les médecines traditionnelles, alternatives et complémentaires pour la société contemporaine » (Developing Traditional, Alternative and Complementary Medicines for Today's Society), données et observations recueillies par Évelyne Micollier présente à ce congrès). Ajoutons que Volker Scheid (Chinese Medicine in Contemporary China - Plurality and Synthesis, op. cit.) a formalisé ce concept. 
avec la médecine occidentale, que la médecine chinoise saura retrouver sa légitimité médicale. Ils soulignent que la médecine chinoise contemporaine connaît un besoin urgent d'évolution et de transformation profonde. L'anthropologue chinois Hor Ting explique ainsi ce que pensent spontanément beaucoup de ses compatriotes:

En Chine, en raison de la transformation de la société traditionnelle, la médecine chinoise a été modernisée par rapport à ses aspects traditionnels. Pour la plupart des Chinois contemporains, comme pour moi-même, la modernisation de la médecine chinoise semble complètement naturelle et incontestable. Et ce point de vue repose a priori sur trois présupposés :

La médecine chinoise doit évoluer d'une forme ancienne à une nouvelle ;

La médecine chinoise doit muer d'un niveau actuel primitif à un degré supérieur pour parachever sa métamorphose ;

La pratique non scientifique actuelle de la médecine chinoise doit devenir scientifique ${ }^{(20)}$.

Ensuite, dans une approche critique, Hor Ting démonte lui-même ces présupposés qu'il vient d'énoncer. D'après lui, la médecine chinoise ne devrait pas chercher à se trouver une nouvelle forme parce que, hors de Chine, c'est précisément en tant que médecine « traditionnelle » qu'elle est adoptée par les Occidentaux comme « médecine du futur »(21). De plus, la médecine chinoise constituant « un système bien établi de connaissances et de techniques » représente de facto une « médecine empirico-spéculative, parvenue dans son développement à une complète maturité » et ne doit pas viser de nouvelles métamorphoses pour pouvoir prétendre être une tradition médicale accomplie (22). Enfin, en remarquant que c'est l'efficacité thérapeutique qui compte fondamentalement dans l'acte médical de soigner plutôt que l'explication scientifique qu'on en donne (23), Hor Ting va jusqu'à renverser les perspectives en déclarant que, du point de vue de l'histoire des sciences, c'est plutôt la médecine occidentale qui, en prétendant s'appuyer dans toutes ses démarches sur une « objectivité scientifique sensible » dont elle fait son unique critère de vérité, devrait plutôt être considérée comme étrange : ainsi ce serait la médecine occidentale, et non pas la chinoise, qui représenterait « un cas isolé dans l'histoire de la médecine » (24). Ce raisonnement montre comment la question du statut épistémologique de la médecine chinoise peut indirectement ébranler certains dogmes scientistes et conduire à tort, par excès inverse, à caricaturer la connaissance dite « scientifique » et à engendrer une vision réductrice de la science, négligeant le fait que le modèle du paradigme scientifique consiste en une remise en question perpétuelle des résultats antérieurs par l'insertion de nouveaux et que, en tant que telle, la démarche scientifique ne s'intéresse qu'à ce qu'elle peut connaître en l'état des connaissances du moment. En outre, ce paradigme s'appuie sur une théorie de la connaissance « ouverte » en perpétuelle construction et donc reconstruction. De ce point de vue, les résistances épistémologiques que rencontre la médecine chinoise dans son processus de diffusion sont doubles : d'une part, la remise en question systématique de ses pratiques et de ses résultats exigée de la médecine chinoise par le paradigme scientifique dominant produit des résistances internes à une modification de ses modes de fonctionnement; d'autre part, contrainte de rendre compte de son expérience à partir de résultats expérimentaux quantitativement mesurables, la médecine chinoise se trouve confrontée à un défi externe d'un genre totalement nouveau dans son histoire.

\section{Troisième type d'enjeu : mettre en place des institutions compétentes et des politiques publiques efficaces}

La médecine chinoise n'aurait pas aujourd'hui en Chine et dans le monde le statut, même épistémologiquement flou et idéologiquement discuté qui est le sien, sans l'appui politique majeur que les autorités de RPC ont choisi stratégiquement de lui donner à la fin des années 1950.

Le fait que la Chine ait mis en place, à partir de la fin des années 1950, de puissants organismes institutionnels chargés d'assurer la diffusion nationale et internationale de la médecine chinoise est incontestable (25). De plus, avec Kim Taylor, « il est aussi permis de remarquer que c'est seulement après que Hong Kong fut revenu dans le giron de la Chine après 1997, que des mesures similaires (à celles qui eurent lieu entre 1953 et 1956 en Chine) furent prises pour institutionnaliser et standardiser (la médecine chinoise) (26) ».

Constatons, pour le moins, que ce processus s'est intensifié après la rétrocession de Hong Kong. Un tel soutien politique fort de la part des autorités de la RPC envers la médecine chinoise n'autorise cependant ni à la réduire à une médecine politique ni à en déduire que l'intérêt de « l'Occident » fut éveillé davantage par les aspects « traditionnels » de la médecine chinoise plutôt que par ses « mérites scientifiques »(27). En effet, si le caractère politique de la MTC est souvent décrié, celui-ci n'enlève rien à ses mérites scientifiques et surtout thérapeutiques.

Pour espérer comprendre comment le gouvernement chinois structure cette discipline, ou quelles sont les lignes politiques définies par lui, il importe de connaître les principales institutions mobilisées par lui pour la promouvoir et œuvrer à sa diffusion institutionnelle. Or, aujourd'hui, l'organisme administratif central dans le développement de la MTC en Chine et dans le monde est la State Administration of Traditionnal Chinese Medicine of the People's Republic of China (中華人民共和國-國家中醫藥管理局 - SATCM-PRC) (28) qui est chargée de dix missions essentielles. Avec l'aide des membres du vaste réseau qu'elle dirige dans les diverses institutions chinoises en Chine et dans le monde, cette institution doit :

1) Proposer des stratégies, des plans et des politiques aptes à développer la MTC et l'usage des médecines ethniques ;

2) Superviser toutes les applications concrètes de la MTC, tant préventives que cliniques;

3) Coordonner l'intégration entre la MTC et la médecine occidentale ;

4) Guider l'exploration, la synthèse et l'amélioration de la formulation théorique de la MTC;

20. Ting Hor, « Modernisation of Chinese Medicine: An Anthropologist's View », in Ping-Chung Leung (et al.), A comprehensive guide to Chinese medicine, Singapour, World Scientific, ch. 12, p. 301-323.

21. Ibid., p. 304.

22. Ibid., p. 307.

23. Ibid., p. 311.

24. Ibid., p. 310 .

25. Voir à ce sujet l'étude détaillée de Wen Xiang, Minguo zhongyi yizheng (La politique médicale de la médecine chinoise en République populaire de Chine), Pékin, Zhongguo Zhongyiyao chubanshe, 2007.

26. Kim Taylor, Chinese Medicine in Early Communist China, 1945 - 1963. A Medicine of Revolution, op. cit., p. 87 .

27. Ibid., p. 109.

28. Nous utiliserons volontairement ci-dessous ce sigle "SATCM-PRC » plutôt que celui de « SATCMC », utilisé habituellement dans les traductions officielles (cf. la note 19 ci-dessus), afin de souligner le caractère politique du terme : la Chine n'étant pas constituée que de la RPC, cette nuance pourrait s'avérer, dans certains contextes, aussi maladroite que celle de zhongyi par « MTC ». 
5) Diriger la protection, l'exploration et l'utilisation rationnelle à l'échelle industrielle de la pharmacopée chinoise ;

6) Promouvoir les actions éducatives à tout niveau pour assurer le renouvellement d'un vivier de compétences en MTC ;

7) Diriger les recherches scientifiques et techniques susceptibles d'améliorer le crédit accordé à la MTC;

8) Assurer la protection du patrimoine immatériel que constitue la MTC tant au niveau diagnostique et thérapeutique que littéraire et culturel ;

9) Entretenir les collaborations internationales visant à une meilleure diffusion de la MTC dans le monde, spécialement en partenariat avec Hong Kong, Macao et Taiwan ;

10) Et enfin - last but not least! - « accomplir toute les tâches demandées en ce domaine par le Conseil d'État et le ministère de la Santé chinois $\gg(29)$.

Un secrétaire général et cinq vice-commissaires adjoints président cette administration. La carrière de ces grands fonctionnaires de la médecine chinoise éclaire le caractère politique de cette institution et la diversité de leurs origines témoigne de sa dimension nationale, dans le respect de l'équilibre entre régions ${ }^{(30)}$. Il importe de souligner également qu'aucun d'eux n'a une formation en biomédecine mais que tous ont, par contre, un diplôme supérieur en médecine chinoise (31). Ces deux détails permettent de souligner combien la SATCM-PRC peut révéler à la fois un caractère national très marqué et constitue une forme d'organisation atypique par rapport aux institutions occidentales habituelles où les décisions politiques concernant les questions de santé sont souvent confiées essentiellement à des experts formés en biomédecine.

Tous ces fonctionnaires sont au service d'une même mission : promouvoir la médecine chinoise en Chine et dans le monde selon les souhaits et dans le respect des instructions reçues des autorités de la RPC. De nombreuses autres associations en Chine travaillent dans cette direction mais toujours en relation avec cette autorité centrale de la SATCM-PRC. On mentionnera parmi elles la « confédération pour l'universalisation des médicaments chinois» (中藥全球化聯盟 - Zhongyao quanqiuhua lianmeng) qui fut fondée en 2003 et vise à rassembler des autorités politiques du monde entier, des universités, des centres de recherche et des industries pharmaceutiques pour promouvoir la modernisation de la médecine chinoise, assurer la protection de son capital culturel, améliorer la qualité de ses produits, en créant une plate-forme d'échanges sur ces questions, incitant à la recherche hospitalière, et permettre à la tradition de la pharmacologie et de la médecine chinoise d'entrer dans le courant dominant des études médicales. Depuis sa fondation, cette confédération a déjà organisé neuf conférences académiques dont la dernière en août 2011 à Shanghai (32).

L'utilité de la SATCM-PRC ne s'est pas limitée à la Chine ni même à la médecine chinoise mais a contribué également à faire reconnaître au niveau international la nécessité de mettre en place, pour la gestion politique des médecines alternatives, des institutions adéquates distinctes de celles qui s'occupent directement du domaine de la biomédecine ${ }^{(33)}$. En revanche, cet organisme chinois ne connaît pas d'équivalent dans la plupart des autres pays. Dans la poursuite de la reconnaissance internationale de la médecine « chinoise » comme appartenant au patrimoine « mondial » de l'humanité, il serait opportun de réfléchir à des SATCM nationales ou éventuellement supranationales (comme par exemple une SATCM-Europe) qui n'émaneraient pas directement de la SATCM-PRC mais qui seraient créées en s'inspirant du modèle de cette institution. La Corée du Sud s'est, par exemple, engagée dans cette direction en créant dans les années 1990 une série d'institutions destinées à promouvoir sa propre tradition médicale (34). La réponse que les autorités publiques d'un pays ou d'un lieu peuvent apporter aux besoins de thérapeutes ayant recours à la médecine chinoise dans leurs pratiques médicales (autant qu'à ceux des malades faisant appel à eux)

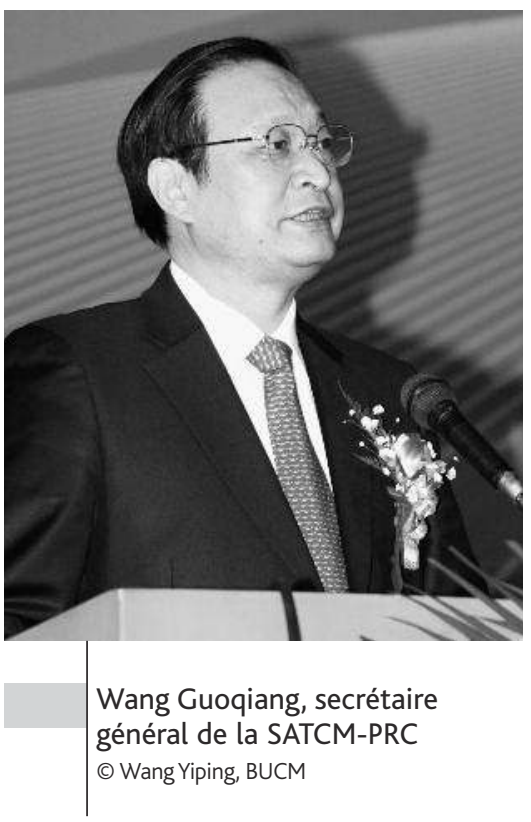
passe par la mise en place de structures politiques adaptées à cette discipline et à ce système médical spécifique. L'expérience institutionnelle chinoise originale que constitue l'expérience de la SATCM-PRC, chargée de maintenir vivant un système médical complémentaire au système dominant de la biomédecine, pourrait, à juste titre, inspirer dans le monde d'autres initiatives similaires ${ }^{(35)}$. Les décisions de savoir qui, dans un pays donné, peut parler de médecine chinoise en connaissance de cause, coordonner des formations dans cette discipline, statuer sur les compétences nécessaires à son exercice, orienter les recherches dans ce domaine sont des questions vitales pour l'avenir de la médecine chinoise dans le monde et qui ne sont ni idéologiques ou philosophiques mais politiques et économiques.

29. La version anglaise : www.satcm.gov.cn/English2010/structure/2010-10-06/150.html

30. Le secrétaire général, Mr. Wang Guoqiang (王國強), est né en 1955 à Shanghai dans une famille originaire de l'Anhui. Après avoir travaillé en Mongolie intérieure, il entra au PCC en 1973 puis étudia la MCT à Pékin où il fut diplômé en 1978. II travailla ensuite, à partir de 1983, dans la Commission nationale responsable de la planification familiale où il resta actif jusqu'en janvier 2007, date à laquelle il devint vice-ministre de la Santé et secrétaire responsable de la SATCM-PRC.

31. Les cinq commissaires adjoints sont : 1) Wu Gang (吳剛), né en 1953 dans le Shanxi, diplômé au collège de MC de Pékin en 1976 ; 2) Yu Wenming (于文明), né en 1963 au Shandong, diplômé dans le cadre de l'institut de MC du Shandong ; 3) Li Daning (李大寧), né en 1953 dans la province du jilin, diplômé en MC à l'institut de MC du Heilongjiang ; 4) Ma Jianzhong (馬建中), né à Zhengzhou au Henan en 1957, diplômé au collège de MC du Henan en 1976 ; 5) Wang Zhiyong (王志勇), né en 1966 dans le Shanxi, diplômé en 1989 de l'institut de MC de Pékin.

32. www.zhong-yao.net/new/zy/201109/249110.html

33. La définition des « médecines traditionnelles » embrasse un large éventail de thérapies, de pratiques et de savoirs très divers selon les pays et les localités. Dans le domaine de la santé publique, elles sont conceptualisées comme des « médecines alternatives » ou « médecines complémentaires » (CAM « Complementary and Alternative Medicine). Or, les mesures de politique publique prises par la Chine et sa volonté politique à long terme de promouvoir la médecine traditionnelle rencontrent les préoccupations des États et d'acteurs civiques et économiques à une échelle internationale ainsi que les recommandations de l'OMS visant à intégrer des médecines traditionnelles dans les systèmes de santé publique. Il y a 30 ans à la Conférence internationale sur les soins de santé primaire d'Alma-Ata (1978), le système chinois était apparu comme un modèle à imiter pour les autres États en particulier ceux dont les ressources médicales étaient très limitées avec, à l'horizon, un objectif de « santé pour tous en l'an 2000 ». La déclaration de Pékin adoptée à l'issue du premier congrès mondial de l'OMS sur la médecine traditionnelle (7-9 novembre 2008) et destinée notamment aux États qui n'auraient pas encore œuvré pour intégrer les médecines traditionnelles appelle à une application plus généralisée des mesures de politique publique et des stratégies de l'OMS pour la médecine traditionnelle (2002-2005) (Évelyne Micollier, « Cestion de l'épidémie et usages de la médecine chinoise. Traitements et recherche en médecine chinoise traditionnelle dans le contexte de l'extension du traitement médical et social du SIDA en Chine », art. cit., p.76).

34. Voir l'article de Kang Yeonseok dans ce dossier.

35. Concernant le terme de biomédecine, voir Anne Guillou et Évelyne Micollier, « Anthropologie et santé en Asie du Sud-Est : dynamiques et courants de recherche », Moussons. Revue de recherche en sciences humaines sur l'Asie du Sud-Est, n 15, numéro thématique Santé, 2010, p. 177-193, p. 187 note 3. 


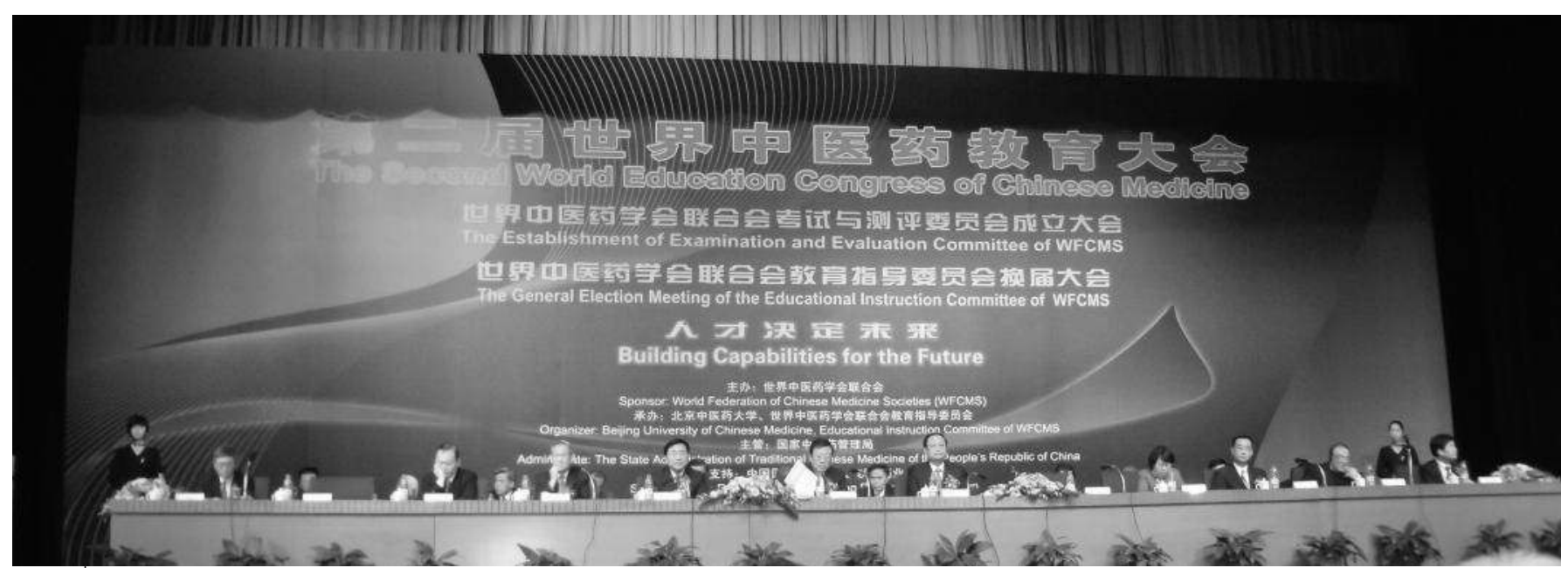

Le Second Congrès pour l'éducation mondiale de la médecine chinoise s'est tenu à Pékin entre les 28 et 30 Octobre 2011. Des responsables du ministère de la Santé et de la SATCM-PRC président ce congrès auquel participaient 500 personnes dont une centaine venues de 31 pays étrangers @ Pierre-Henry de Bruyn

\section{Quatrième type d'enjeu : trouver les moyens économiques de son développement}

Le fait que la médecine chinoise représente une source potentielle d'importants bénéfices financiers donne à son organisation une dimension économique qui complique encore le processus de sa diffusion. Sans parler des questions de formation (que nous aborderons séparément au point suivant), plusieurs aspects sont à souligner en ce domaine : le marché des médicaments, les débouchés professionnels, le remboursement des soins et le financement de la recherche. Nous évoquerons principalement la situation en Chine.

\section{Le marché des médicaments}

Les enjeux économiques du marché des médicaments chinois représentent l'intérêt financier le plus évident. Ils influencent de manière décisive les discours actuels sur la médecine chinoise en Chine autant qu'à l'étranger. Avec quelques 6000 fabricants de médicaments en Chine, capables de produire plus de 1000 médicaments chimiques et 8000 médicaments traditionnels chinois, les personnes impliquées dans la production de publicités autour de ces produits sont très nombreuses : le Journal de la médecine chinoise en Chine comptait déjà en 1999, quelques 1000 usines de médicaments dont 178 de taille moyenne ou grande avec une production totale de plus de 23,5 milliards de dollars américains (194,6 milliards de RMB) (36). Or, ce chiffre d'affaires a encore considérablement augmenté ces dix dernières années. En 2007, l'estimation des ventes de produits pharmaceutiques traditionnels s'élevait en Chine à 5,5 milliards de dollars US sur un total de 22,6 milliards tous médicaments confondus ${ }^{(37)}$. La dimension économique sous-jacente aux discours actuels des autorités chinoises cherchant à promouvoir la diffusion de la MTC est omniprésente. Le rapport de mars 2008 rédigé par l'Institut de médecine chinoise de l'université chinoise de Hong Kong (ICM) sur la demande des autorités politiques de Hong Kong mentionne par exemple que :

La médecine chinoise jouit naturellement d'un certain prestige dans le champ des médecines traditionnelles [...] Une preuve du renouveau sont les dépenses croissantes faites en Europe et aux États-
Unis dans le domaine des médecines traditionnelles et alternatives. Le potentiel économique de la médecine chinoise est par conséquent international.

[...] Si les produits de la pharmacopée traditionnelle peuvent être prouvés cliniquement efficaces, le potentiel économique en sera considérablement multiplié.

[...] Hong Kong peut développer avantageusement sa compétitivité dans les produits de la médecine chinoise et utiliser cette nouvelle donne pour poursuivre plus loin sa croissance économique(38).

Ce même rapport mentionne cependant que l'industrie de médecine chinoise de Hong Kong doit également faire face à de nombreuses difficultés : d'abord, la qualité des plantes n'y est pas assez sûre ; ensuite, les laboratoires responsables du contrôle de qualité sont jugés déficients ; et enfin, les techniciens compétents ne sont pas en nombre suffisant ${ }^{(39)}$. Plus loin, il mentionne encore que les industries locales de médicaments en médecine chinoise manquent d'investissement autant que d'esprit d'innovation ${ }^{(40)}$. Cet exemple des questions économiques qui se posent à Hong Kong dans une réflexion sur l'état de la diffusion de la médecine chinoise témoigne combien celles-ci sont importantes.

\section{La question des débouchés professionnels}

À côté des aspects proprement industriels, il faut ajouter les dimensions liées à la problématique des débouchés professionnels pour les

36. Chiffres donnés par Ping-Chung Leung, " The State of Chinese Medicine in China Today 》, in A comprehensive guide to Chinese medicine, op. cit. p. 205-207.

37. Voir Catherine Legrand « Les opportunités d'un marché complexe », Connexions 45, mai-juin 2008, p.51.

38. Cf. Ping-Chung Leung (principal investigator), Developing Hong Kong into a Chinese Medicine International Platform - A Careful Study, Hong Kong, Institute of Chinese Medicine (CUHK), 2008, p. xx (cité ci-dessous sous le sigle : DHKCMIP-08). L'intérêt de ce document tient au fait que l'information qu'il contient repose sur une série d'enquêtes menées non seulement à Hong Kong mais aussi en Chine. En effet, le vice-président de l'université de médecine chinoise de Guangzhou (廣州中醫藥大學), Wang Ningsheng 王寧生 a dirigé pour ce rapport une vaste recherche qui porta sur la province de Canton ainsi que Nankin, Shanghai, Pékin et même la province du Heilongjiang (p. 17). En 2006, la commission de la médecine chinoise du comité d'hygiène du Yuan exécutif de Taiwan (Taiwan xingzhengyuan weishengshu zhongyi zeiyuanhui) avait rédigé un document analogue intitulé : Visions on Chinese Medicine in Taiwan.

39. DHKCMIP-08, p. xxiv.

40. Ibid., p. xxxi. 
personnes formées dans ce système médical. Ce problème se pose évidemment partout dans le monde de façon très différente qu'en Chine continentale. Dans l'enquête sur l'état de la médecine chinoise en RPC dirigée par le professeur Wang Ningsheng (王寧生) de l'université de médecine chinoise de Canton, il est souligné que :

La situation de la médecine chinoise (en RPC), du point de vue thérapeutique, se distingue clairement par des caractéristiques et une situation exceptionnelles. Actuellement dans l'ensemble du pays, il y a 3072 hôpitaux de médecine chinoise de niveau urbain ou supérieur, avec 332000 lits dans 161 spécialités différentes. Dans 90 \% des hôpitaux de médecine occidentale il y a des départements de médecine chinoise. 524000 personnes ont une qualification diplômante en médecine chinoise (中醫資格證書 - zhongyi zige zhengshu). 237000 sont enregistrés comme maître en médecine chinoise (註冊 中醫師 - zhuce zhongyishi). Le nombre de consultations annuelles enregistrées en médecine chinoise est de 244 millions (2,44 億次 yici), soit $18,49 \%$ du nombre total de consultations ${ }^{(41)}$.

La question des débouchés professionnels de personnes formées à la médecine chinoise semble par conséquent moins délicate en Chine que dans la plupart des autres parties du monde. Cependant, là aussi le problème demeure pour deux raisons : d'une part, les soins en médecine chinoise, étant moins coûteux que ceux de la médecine occidentale, rapportent aussi proportionnellement moins d'argent à ceux qui les procurent; d'autre part, le statut social de ceux qui pratiquent ce genre de médecine souffrant de la réputation « non-scientifique » de cette discipline, leur reconnaissance sociale tend à s'affaiblir. Le professeur Wang Ningsheng note ainsi que « le soutien populaire de la médecine chinoise tend de plus en plus à se relâcher et à s'affaiblir 》 (中醫的民間基礎越來越鬆散薄弱 - Zhongyi de minjian jichu yuelai yue songsan boruo).

\section{La politique de remboursement}

Le troisième facteur économique susceptible d'influencer de façon majeure le développement de la médecine chinoise est la politique de remboursement des soins procurés, (consultations, ordonnances, services, produits...). En RPC, le prix des consultations en médecine chinoise est remboursé de façon similaire à ce qui est pratiqué pour celles en biomédecine. À Singapour, cet acte est couvert par l'assurance médicale, même dans les cliniques privées, tandis qu'à Taiwan, le degré de remboursement varie selon les assurances.

Les arguments bien connus en faveur du choix de la médecine chinoise par les patients sont les suivants : une moindre probabilité d'effets secondaires et un coût moins élevé à l'exception des patients qui recherchent la meilleure qualité de produits et de services ${ }^{(42)}$. En conséquence, le coût est également moins onéreux pour les assurances maladie quelles qu'elles soient. Si nous devons comparer concrètement les coûts, il suffit de conduire des observations au guichet de la plupart des hôpitaux dans les grandes villes chinoises qui vendent des produits des deux médecines : en effet, tous les patients qui ont une ordonnance passent par ces guichets. Produits traditionnels et biomédicaux sont souvent mais pas toujours fournis séparément dans deux pharmacies de l'hôpital situées l'une à côté de l'autre. Le personnel est constitué en général de pharmaciens ou d'assistants spécialisés. Pour une pathologie bénigne commune, moins de 10
RMB permettent de se procurer des produits ordinaires de la pharmacopée traditionnelle alors que par exemple une boîte de paracétamol coûte environ 20 RMB et la boîte d'antibiotiques le double ${ }^{(43)}$. Précisons que le secteur hospitalier est le principal fournisseur de médicaments en Chine à hauteur d'environ $80 \%$ du marché total. Dans les années 2000, les pharmacies fonctionnant hors du système hospitalier ont développé le marché lucratif des produits « OTC » (over the counter) ${ }^{(44)}$ pour accroître leur part sur le marché intérieur : elles vendent donc un certain nombre de produits traditionnels qui bénéficient du label OTC.

Cela dit, il faut, pour ce qui est de la Chine ${ }^{(45)}$, tout de même limiter l'impact de la politique de remboursement des soins sur l'usage et le recours à la médecine chinoise dans la mesure où seulement une petite minorité (20\%) de la population chinoise avait une assurance maladie au début des années $2000^{(46)}$ et, selon les sources, on estime que $15 \%$ à $20 \%$ de la population bénéficiait alors de 60 à $80 \%$ des ressources médicales publiques. Les catégories sociales privilégiées étaient principalement les employés du gouvernement et ceux des entreprises d'État. $50 \%$ de la population urbaine et $80 \%$ de la population rurale n'avaient aucune couverture médicale en $2000 ; 50 \%$ des habitants des zones rurales n'avaient pas accès aux soins à cause de leur coût (OMS, fiche pays, 2000). La détérioration des infrastructures, le manque de personnel, et une prévalence accrue de certaines pathologies étaient attestés dans la majorité des zones rurales ${ }^{(47)}$. En deux décennies, le système chinois de soins de santé primaire, pourtant considéré comme le modèle à suivre pour les pays en développement par l'OMS (1978, déclaration de la Conférence d'Alma-Alta), avait complètement disparu au profit d'un système commercialisé et de facto privatisé qui s'appuie sur des structures sanitaires publiques contraintes à s'autofinancer. La réforme de la santé officiellement lancée en $2009{ }^{(48)}$ a pour objectif principal de réduire les inégalités face à la santé et donc la pauvreté ${ }^{(49)}$. Rendues publiques en 2008 et 2009 , ses lignes directrices pré-

41. Ce rapport dirigé par Wang Ningsheng est publié en annexe 1 du rapport de 2008 ci-dessus mentionné (DHKCMIP-08) sous le titre « Neidi zhongyiyao fazhan diaoyan baogao » (Rapport de recherche par enquêtes sur le développement de la médecine chinoise en Chine), p. S6, 3.1.

42. Dans ce cas, le coût du traitement peut largement dépasser celui de génériques de produits biomédicaux fabriqués par les entreprises chinoises.

43. Observations effectuées par Évelyne Micollier à Pékin et à Canton en 2007-2008.

44. Label international octroyé à des produits qui peuvent être vendus sans ordonnance destinés à traiter des pathologies bénignes. Des réglementations locales et/ou nationales s'appliquent pour l'attribution du label.

45. Le paragraphe qui suit est adapté de l'article d'Évelyne Micollier « Pourquoi une réforme du système de santé est-elle urgente ? » dans Fabrice Duléry (éd.), Aujourd'hui la Chine, CRDP académie de Montpellier et CNDP (Centre National de Documentation Pédagogique), Montpellier, 2011, p. 88-90.

46. Le système de soin antérieur à l'ère de la Réforme (1979- ) devait être très rudimentaire mais il avait le mérite d'exister, d'être plus égalitaire avec environ $90 \%$ de la population qui bénéficiait d'une quasigratuité des soins grâce des subventions nationales, provinciales ou décentralisées à une échelle administrative plus locale. Selon un rapport de la Banque Mondiale des années 1990 (World Bank, 1997, China 2020 ; Financing Health Care, Washington D.C., World Bank, p. 1), en 1975, la prise en charge médicale était assurée par le système de coopérative rurale, le gouvernement et les entreprises d'Etat et bénéficiait à près de $90 \%$ de la population (la population urbaine dans sa quasi-totalité et $85 \%$ de la population rurale).

47. Voir Marilyn Beach, « China's rural health care gradually worsens », The Lancet, 2001, vol. 358, p. 567.

48. Chen Zhu, «The Launch of the health-care reform plan in China », The Lancet, 2009, vol. 373, p. 1322-1324.

49. Elle fait déjà l'objet de nombreuses publications car elle était très attendue par les acteurs nationaux et internationaux du développement (voir Challenges for China's Public Spending: Towards Greater Effectiveness and Equity, OECD Study, 2006). Parmi elles, voir la remarquable collection d'articles sur la santé en général et la réforme en particulier publiée en ligne dans la prestigieuse revue The Lancet, 2008 et 2009, www.thelancet.com ; bulletins de l'OMS dont Hu Shanlian « Universal Coverage and health financing from China's perspective », Bulletin of the World Health Organization, 2008, vol. 86, $n^{\circ} 11$, p. 819 ; Parry J., Cui Weiyuan « Making health care affordable in China », Bulletin of the World Health Organization, 2008 , vol. 86, n 11, p. 821-823; sur les multiples défis posés par la réforme et le débat interne qu'elle suscite, cf. Lu Xiaoging, Charles W. Freeman, China's Capacity to Manage Infectious Diseases: Global Implications, Center for Strategic and International Studies, mars 2009, 101 p., chap. 5 et 6. 
voient la généralisation d'un système d'assurance maladie avec une couverture de base pour $90 \%$ de la population en 2011, l'amélioration de l'accès aux soins avec une réforme hospitalière au cœur du projet, enfin un financement conséquent et la fourniture en médicaments destinés aux hôpitaux pour endiguer les dérives d'une commercialisation excessive. Cette réforme aura donc a priori une influence considérable sur le développement de la médecine chinoise parce qu'elle facilitera en principe l'accès aux soins médicaux pour une majorité de la population dans le système de santé publique donc a fortiori en médecine chinoise.

\section{Le financement de la recherche}

Enfin, le dernier facteur économique décisif est celui du financement de la recherche en médecine chinoise. Il existe deux dangers ici, l'un extrinsèque et l'autre interne à la discipline : d'une part, en favorisant la recherche par exemple des « éléments actifs » de la pharmacopée chinoise, la pression des grands groupes pharmaceutiques (étrangers ou chinois) risque de pousser progressivement les travaux dans ce domaine à évoluer trop unilatéralement dans une direction étrangère et même destructrice de dimensions fondamentales et constitutives de l'héritage culturel spécifique qui constitue la médecine chinoise ${ }^{(50)}$; d'autre part, la médecine chinoise, qui provient de l'accumulation historique de nombreuses expériences thérapeutiques, peine encore à élaborer des processus d'expérimentation et de validation spécifiques universellement admis par la communauté scientifique.

En Chine, la recherche en médecine chinoise est principalement financée dans le cadre des programmes de recherche et développement des groupes pharmaceutiques chinois. Certains groupes produisent et commercialisent exclusivement des produits de médecine chinoise ; d'autres entreprises ne sont pas spécialisées et financent aussi la recherche en biomédecine et en médecine intégrée. Les protocoles de recherche biomédicaux peuvent intégrer des éléments qui relèvent de la médecine chinoise et inversement ceux de médecine chinoise des éléments de biomédecine. Les organismes d'État tels que le ministère de la Santé, celui de la Science et de la technologie et celui de la Culture et du patrimoine contribuent également au développement de la recherche en médecine chinoise. Dans les années 2000, le gouvernement a accru son soutien financier de manière conséquente. Dans ces recherches en milieu industriel, universitaire ou hospitalier, une même méthodologie scientifique tend à être appliquée.

Au cœur d'enjeux économiques complexes, la recherche et le développement en médecine chinoise, par son potentiel de mise sur le marché de produits de pharmacothérapie (中醫藥 - zhongyiyao) et la pharmacologie traditionnelle (中醫藥學 - zhongyiyaoxue), s'insèrent dans le cadre du marché mondial de la santé. Ces produits constituent une niche de marché bienvenue pour l'industrie pharmaceutique chinoise qui comprend une majorité d'entreprises publiques. Suite aux contraintes imposées à la Chine lors de son entrée à l'OMC (2001), ces entreprises ont dû changer rapidement de stratégie car elles ne pouvaient plus fabriquer systématiquement les génériques de produits biomédicaux brevetés dans les pays développés qui constituaient pourtant la quasi-totalité de leur production. Des négociations pour le développement de recherches internationales en médecine chinoise, attestent que la stratégie de l'industrie et des autorités chinoises vise à obtenir l'AMM (autorisation de mise sur le marché) dans les pays développés par un enregistrement des complexes de la pharmacopée. L'objectif est de parvenir à entrer dans le marché par l'inscription dans la catégorie médicaments plutôt que dans celle de compléments alimen- taires. Or le cadre juridique et les normes de validation scientifique sont beaucoup plus contraignants pour les médicaments : ces normes prescrivent le recours au modèle EBM (Evidence-Based Medicine) et la réalisation d'essais cliniques concluants. Une AMM « aménagée » est prévue dans le cadre réglementaire de l'Agence européenne de médecine pour les médecines non conventionnelles : elle concerne (1) les médicaments d'usage médical bien établi c'est-à-dire pour lesquels les preuves d'innocuité et d'efficacité, bibliographiques ou expérimentales, ont été apportées ; (2) les médicaments « traditionnels » pour lesquels une « longue » tradition d'usage (30 ans minimum dont 15 dans la communauté européenne) est reconnue (Directive 2004/24/EC). De nombreux projets de recherche scientifique visant à tester des produits de médecine chinoise ont été conduits dans les années 2000 : par exemple, le projet Herbalome, ambitieux et de long terme (15 ans), est présenté dans la revue Science. Ils s'appuient sur des méthodes telles que le criblage de haut niveau des produits, les tests de toxicité et les essais cliniques : l'objectif est d'identifier les substances actives et les substances toxiques dans les complexes de MTC (51). Un autre exemple concerne un appel à projets de l'ANR française (Agence nationale de la recherche) lancé en 2008 dans le cadre du programme de biotechnologies pour la santé où la médecine chinoise est explicitement mentionnée. L'appel est révélateur de politiques scientifiques en amont pour encourager le développement de la recherche scientifique dans le cadre d'un partenariat franco-chinois de recherche publique (52). Force est de constater pourtant qu'en dépit d'un effort politique bilatéral, aucun projet n'a finalement été financé dans le cadre de cet appel (53).

\section{Cinquième type d'enjeu : l'innovation d'une didactique spécifique}

La dernière catégorie d'enjeux majeurs que rencontre la diffusion du patrimoine de la médecine chinoise concerne la didactique spécifique de cette discipline. Passée brusquement, durant les années 1950, d'un mode de transmission individualisé et le plus souvent intrafamilial à une structure plus institutionnelle et universitaire, l'enseignement de la médecine chinoise ne s'est pas encore remis, même en Chine, de cette mutation fondamentale. Trois observations l'attestent : le quasi-culte des « Lao Zhongyi » (老中醫 - vénérables médecins ${ }^{(54)}$ ) organisé au niveau local et même national ; la survivance de validation officielle des compétences de parcours effectués avec un maître en médecine chinoise, en dehors de tout parcours institutionnel homologué ; et enfin certains propos d'enseignants de médecine chinoise demandant aujourd'hui en Chine un retour à un enseignement plus classique. Nous nous arrêterons ici uniquement sur ce dernier point (55).

50. Par exemple : le fait que les catégories utilisées dans le diagnostic soient les mêmes que celles employées pour classer les plantes; que les plantes ne soient qu'exceptionnellement données seules mais presque toujours dans le cadre d'une formule (elle-même fruit d'une longue expérience) ; ou encore que l'attribution thérapeutique de ces plantes soit parfois liée au rythme des saisons.

51. Revue Science « TCM under the microscope: Researchers hope the project will lead to better quality control », 12 février 2008.

52. Richard Stone, «Lever le voile sur la médecine traditionnelle chinoise » (Lifting the Veil on TCM), Science, vol. 319, n 5864, 8 février 2008, p. 709-710.

53. Ce long paragraphe emprunte à Évelyne Micollier (« Cestion de l'épidémie et usages de la médecine chinoise. Traitements et recherche en médecine chinoise traditionnelle dans le contexte de l'extension du traitement médical et social du SIDA en Chine », art. cit., p. 82 ; « Un savoir thérapeutique hybride et mobile - Éclairage sur la recherche médicale en médecine chinoise en Chine aujourd'hui », art. cit., 2011, p. 56-57).

54. Le caractère « lao » signifie « vieux » au sens premier mais ici doit plutôt être traduit par « vénérable ».

55. Pour le culte des Lao zhongyi, voir dans ce dossier ce que Éric Marié et Frédéric Obringer expliquent au sujet des tentatives récentes de revalorisation de l'enseignement maître-disciple (師徒傳承 - Shitu chuancheng). 
En Chine, la médecine traditionnelle a longtemps été considérée comme une tradition doublement savante, d'une part parce que sa maîtrise pratique ne pouvait s'acquérir que grâce à une longue expérience personnelle (prise des pouls, diagnostic, modification discernée des formules de pharmacopée selon les besoins de tel ou tel patient etc.), et d'autre part, parce que sa connaissance théorique exigeait une vaste culture littéraire de textes rédigés essentiellement en chinois classique ${ }^{(56)}$. Le défi de trouver des enseignants capables de briller à la fois dans ces deux domaines se pose en Chine aujourd'hui avec force. Des demandes récurrentes d'un retour à un enseignement plus classique se font entendre actuellement dans le monde de la médecine chinoise. Quelques propos du professeur Liu Lihong (劉力紅) illustreront ce point ${ }^{(57)}$.

Par sa formation, Liu Lihong incarne un mélange entre deux traditions pédagogiques. Formé successivement durant neuf années dans diverses institutions universitaires chinoises contemporaines (1978-1983 : étudiant à la faculté de MC du Guangxi ; 1983-1989 : chercheur dans le département des maladies de chaleur [溫病研究室 - wenbing yanjiu shi] dont les trois dernières à Chengdu ; 1989-1992 : doctorat à la faculté de MC de Nanjing sous la direction du professeur Chen Yiren [ 陳亦人 19242004 ]), il revint ensuite au Guangxi pour enseigner les théories fondamentales puis fut encore invité à l'université Qinghua entre 2002 et 2003. Mais durant sa formation, il eut également l'occasion de suivre une formation plus traditionnelle - comme le faisaient en Chine les disciples séjournant chez leur maître pour y être initié par contact quotidien aux pratiques médicales ${ }^{(58)}$.

Dans certains passages de la préface de son livre Réfléchir à la médecine chinoise, Liu Lihong dépeint une situation relativement sombre de la médecine chinoise en Chine ${ }^{(59)}$ :

Que ce soit dans les académies de médecine chinoise ou dans les départements de médecine chinoise des académies de médecine occidentale, la médecine chinoise est presque devenue une parure. Ceux qui étudient la médecine chinoise n'ont pas confiance en elle ; dès qu'ils rencontrent un peu de difficultés, soit ils administrent en hâte des médicaments occidentaux, soit ils ajoutent aux remèdes habituels de la médecine occidentale un peu de médecine chinoise pour faire bien [...]. Constater que la médecine chinoise soit tombée si bas ne peut que conduire à la morosité(60).

Il fait remarquer que :

Ces dix dernières années, une question souvent soulevée dans le monde de la médecine chinoise est de savoir pourquoi la théorie de la médecine chinoise s'est arrêtée aux problèmes cliniques. Dans n'importe quelle discipline scientifique, c'est la théorie qui marche en tête tandis que la pratique suit lentement derrière(61).

Mais contrairement à cette idée d'une médecine chinoise dénuée de fondement théorique spécifique, Liu Lihong précise sa position personnelle sur ce sujet en écrivant :

Ma façon de voir n'est pas du tout celle-là et se situe au contraire juste à l'opposé : la théorie [de la médecine chinoise] non seulement n'a pas régressé (沒有落後 - meiyou luohou), mais dans de nombreux domaines, elle a même fait d'énormes progrès (大大的超前 - dada de chaogian).

Pour lui, un important problème est didactique :
Il y a actuellement en médecine chinoise un étrange et même effrayant phénomène qui consiste en un affaiblissement progressif de l'enseignement des classiques de la médecine chinoise. La plupart des académies de médecine chinoise contemporaines ont déjà transformé [l'étude] des classiques en un cours (facultatif) à option - celles de Chengdu ou Nanjing, honorables institutions qui autrefois donnaient tant de poids à cette matière ne faisant pas exception. Mais ce genre de transformation constitue-t-il véritablement un progrès ? J'en doute très fortement ${ }^{(62)}$.

Selon Liu Lihong, l'étude des classiques est un élément incontournable de l'apprentissage de la médecine chinoise :

Les classiques sont, à vie, [pour le praticien de médecine chinoise] un cours obligatoire ; si vous voulez vraiment étudier la médecine chinoise, travailler profondément les classiques est le genre de projet que vous devez nécessairement faire ${ }^{(63)}$.

Cette étude est doublement difficile. D'abord d'un point de vue littéraire, l'étude des classiques de la médecine chinoise demande une intelligence philologique élevée permettant d'apprendre progressivement à saisir les multiples sens d'un texte et de pénétrer le sens des mots (字義 - ziyi),

56. La difficulté de trouver et de former au moins quelques spécialistes capables d'assimiler une connaissance non seulement pratique (facultés de médecine) « ou » littéraire (facultés de sinologie) de cette tradition mais plutôt de faire l'unité entre ces deux aspects se pose évidemment de façon encore plus accrue en Occident qu'en Chine. La France a une longue tradition d'innovation dans ce domaine. Déjà en 1813, le docteur Abel Rémusat (1788-1832) présentait une thèse sur la médecine chinoise (Dissertatio de glossosemeiotice sive de signis morborum quae e lingua sumuntur, praesertim apud sinenses). L'année suivante, il fut nommé à la chaire de langue et littérature chinoises et tartares-mandchoues au Collège de France. Plus tard, dans les années 1970, étaient inaugurées à Paris les études taoïstes dans le monde occidental autour des travaux de Kristofer Schipper. Aujourd'hui, deux siècles après la thèse d'Abel Rémusat, pourquoi ne pas envisager la création d'une chaire française ou européenne de médecine chinoise destinée à promouvoir en France et en Europe la protection, et donc la transmission, de ce patrimoine culturel devenu désormais celui de l'humanité ?

57. Né en 1958, Liu Lihong devint en 1992 le premier détenteur d'un doctorat en MTC de la province de Guangxi. Depuis 1997, il est enseignant à l'université de MTC de cette région autonome et s'est spécialisé dans l'usage de formules classiques pour le traitement de maladies persistantes. Grand spécialiste du Shanghan lun (Traité sur les diverses maladies résultants d'une atteinte du froid), célèbre classique de la médecine chinoise rédigé au Ile siècle par Zhang Zhongjing (張仲景; 150-219), Liu Lihong s'est rendu célèbre par la publication de deux ouvrages : Kaiqi zhongyi zhi men (Ouvrir les portes de la médecine chinoise, 1998) et surtout Sikao zhongyi (Réfléchir à la médecine chinoise, 2004) qui se présente comme un commentaire savant du Shanghan lun. À la lecture de ce dernier livre, un des plus célèbres médecins chinois contemporains, le professeur Deng Tietao (燈鐵濤 né en 1916) aurait déclaré : « Désormais, je ne suis plus seul sur mon chemin, il y a quelqu'un derrière désireux de continuer la tradition de la MTC... » (吾道不孤, 後繼有人矣 - wu dao bu gu, hou ji you ren yi). La conversation entre Liu Lihong et Wang Qingyu sur la nature de la transmission nécessaire en médecine chinoise (www.classicalchinesemedicine.org/2011/01/3050/) et la profonde conviction de Liu Lihong sur l'importante primordiale du rôle de l'émotion (感動 - gandong) dans ce processus (www.classicalchinese medicine.org/2011/02/liu-lihong-reflections-on-gandong-real-transmission-of-knowledge-requiresthat-our-hearts-are-moved/) montrent combien la question du mode de transfert du savoir est centrale à ses yeux dans sa réflexion sur l'avenir de la MTC.

58. Liu Lihong fréquenta ainsi durant sept ans Li Yangbo (李陽波 1947-1991), à qui il reconnaît devoir l'essentiel de son premier ouvrage, dont deux années où il vécut chez ce dernier ainsi que d'autres maîtres comme Wang Qingyu (王慶餘 1932-2002) ou Zeng Basheng (鄫矔生) (www.hunyuan.org/ main_html.php? page=qanda\&subpage=24) qui lui enseignèrent le taoisme, ou chez certains médecins comme en 2004 auprès du docteur Li Ke (李可), un vieux médecin travaillant au Shanxi parfois appelé Li Lao (李老, le vieux Li ), ou en 2006 aux côtés de Lu Chonghan (盧崇漢) à Chengdu, grand maître de la troisième génération de l'école de tradition médicale appelée huoshen pai (火神派, courant du feu spirituel) fondée par Zheng Qin'an (鄭欽安 1804-1901) à la fin du XIXe siècle.

59. Liu Lihong, Sikao zhongyi - dui ziran yu shengming de shijian jiedu (Réfléchir à la médecine chinoise - lecture explicative de la temporalité naturelle et biologique), Taipei, Jimu wenhua chuban, 2004 (2009), p. 1.

60. Ibid., p. 14

61. Ibid., p. 15

62. Ibid., p. 18

63. Ibid., p. 70. 
celui des phrases (句義 - juyi) ainsi que la signification globale (總義 zongyi) du texte ${ }^{(64)}$. Ensuite, ce processus demande aussi de cultiver en soi un état d'esprit de réceptivité confiante par rapport au texte qui n'est pas évidente. Liu Lihong fait ainsi remarquer :

Un autre problème auquel il faut prêter attention pour parvenir à bien étudier les classiques [de la médecine chinoise] est qu'il faut remplir une condition fondamentale, ou autrement dit acquérir une qualité essentielle, à savoir celle de savoir entreprendre cette étude dans un état de réceptivité fidèlement respectueuse (信受舉行 xinshou juxing). De nos jours, beaucoup étudient les classiques [de la médecine chinoise] en l'abordant d'un œil critique, considérant que les classiques ne sont pas scientifiques et que, pour cette raison, on serait plus intelligent qu'eux, mais alors, quel classique étudientils encore ? Si vous vous lancez dans l'étude des classiques avec une émotion qui leur est contraire, en les considérant comme dépassés, comment parviendrez-vous à entrer dedans? C'est pourquoi, l'attitude avec laquelle on étudie les classiques est très importante : vous devez complètement les croire, les accepter puis ensuite réfléchir à la façon d'agir conformément à leur esprit ${ }^{(65)}$.

Les propos de Liu Lihong rassemblés ci-dessus ne peuvent évidemment résumer sa pensée profondément nourrie par une étude minutieuse et vivante du Shanghanlun mais témoigne par contre de la force avec laquelle peut être défendue aujourd'hui en Chine par certains un retour à un enseignement de la MTC davantage basé sur les classiques. Cette importance attribuée à l'étude des classiques dans l'apprentissage de la médecine chinoise s'explique par le fait que la pratique médicale hérite d'une longue expérience humaine d'individus se battant depuis des siècles pour maintenir la santé, triompher des maladies et retarder la mort. En s'appuyant principalement sur l'objectivité scientifique et expérimentale des sciences « contemporaines », selon le paradigme scientifique dans lequel elle s'inscrit, la biomédecine tend à considérer ces expériences thérapeutiques du passé comme relativement dépassées par rapport à la force de l'évidence expérimentale ${ }^{(66)}$.

Cette puissance que donne l'habitude d'un recours permanent à l'évidence expérimentale fait la force spécifique de la biomédecine, mais les praticiens de médecine chinoise peuvent parfois la ressentir comme écrasante :

Évidemment, les méthodes pour poser un diagnostic en médecine chinoise et en médecine occidentale sont profondément différentes. La médecine occidentale dispose d'un grand nombre de méthodes modernisées pour l'aider et, de plus, ces méthodes elles-mêmes progressent toujours davantage. Qu'en est-il de la médecine chinoise ? Elle n'a rien de similaire : en tout elle ne peut s'appuyer que sur elle-même. C'est pourquoi étudier la médecine chinoise est plus difficile que d'étudier la médecine occidentale. Si tu étudies la médecine occidentale, toutes les techniques du monde viennent à ton aide : la physique moderne te porte secours; la chimie moderne te soutient ; la biologie moderne t’aide encore davantage. Mais si tu étudies la médecine chinoise, personne ne t'aide et, au contraire, tous semblent vouloir te compliquer la vie en te cherchant noise. C'est pourquoi si quelqu'un veut réussir à étudier la médecine chinoise, et tout spécialement dans le contexte de modernisation actuelle, ce n'est vraiment pas chose facile. Tous devront réfléchir longuement à ce problème et enraciner en eux une confiance inébranlable [en la valeur de la médecine chinoise] ${ }^{(67)}$.

\section{Conclusion :}

L'analyse des principaux types d'enjeux qui polarisent les discussions portant sur la médecine chinoise et qui influent sur son processus de diffusion institutionnelle ne permet pas d'imaginer l'avenir et même la survie de cette tradition médicale sans une certaine inquiétude. Le professeur Xie Peishan (謝培山 - 1934- ) illustrait ce point en disant : « L'avenir de la médecine chinoise se trouve en réalité aujourd'hui ne plus tenir qu'à un fil ! » (68). La réflexion ci-dessus met en évidence quelques points de vigilance qui pourraient éventuellement être utiles pour pouvoir considérer ce futur avec davantage de tranquillité :

1. Prendre conscience de la polarisation idéologique actuelle des débats, tant en Chine qu'en Occident, entre les partisans et les opposants de la médecine chinoise ;

2. Réaffirmer comme centrale la perspective thérapeutique de l'acte médical par rapport au modèle théorique d'approche du corps sur lequel il fonde son efficacité ;

3. Ériger des structures politiques adéquates et de niveau national autant que supranational pour pouvoir assurer une gestion globalisée de l'usage de cette tradition médicale ;

4. Prendre en compte, de façon élargie, les multiples facteurs économiques susceptibles d'influencer l'évolution et la réception de cette tradition ;

5. Encourager les initiatives pédagogiques (respectueuses à la fois de l'histoire de la didactique spécifique à cette discipline et des exigences d'une formation scientifique et critique de niveau international) favorisant un enseignement supérieur de cette tradition médicale ${ }^{(69)}$.

Par ailleurs, il ne faudrait pas sous-estimer le fait que des avantages en faveur de la diffusion institutionnelle d'une certaine médecine chinoise à un niveau global peuvent être identifiés : la taille et l'influence des diasporas chinoises dans toutes les parties du monde, les postes-clés occupés par des cadres chinois à l'OMS, son coût relativement moindre par rapport à la biomédecine pour une diffusion dans les pays en développement, enfin une mobilité internationale accrue des médecins traditionnels et d'une classe moyenne mobile souvent encline à valoriser à l'étranger le patrimoine culturel chinois qui est le sien. Cette mobilité est, bien sûr, rendue possible par le développement économique de la Chine. En complément des enjeux analysés dans cet article, tous ces facteurs contribuent à expliquer que la médecine chinoise est déjà une médecine mondiale. En conséquence, les perspectives de recherches futures qui viseraient à identifier les formes et les transformations de ce savoir, évoluant selon les contextes, sont nombreuses et prometteuses.

64. Ibid., p. 145 .

65. Liu Lihong, Sikao zhongyi - dui ziran yu shengming de shijian jiedu (Réfléchir à la médecine chinoise - lecture explicative de la temporalité naturelle et biologique), op. cit., p. 70.

66. Notons cependant que l'expérience clinique apportée par les études de cas n'est pas non plus un monopole de la biomédecine puisqu'elle est reconnue comme importante par de nombreuses traditions de médecines alternatives.

67. Liu Lihong, Sikao zhongyi - dui ziran yu shengming de shijian jiedu (Réfléchir à la médecine chinoise - lecture explicative de la temporalité naturelle et biologique), p. 127.

68. DHKCMIP-08, p. 18

69. Un rapport officiel rendu aux autorités hongkongaises en 2001 mentionnait l'urgence d'un enseignement de cette tradition lui permettant de s'internationaliser, ce qui impliquerait des cursus définis avec des examens dont la validation soit dûment reconnue (DHKCMIP-08, p. 22). Les trois diplômes universitaires de troisième cycle dédiés à la médecine chinoise qui furent créés ces dernières années à la faculté de médecine de l'université de Montpellier dans le cadre d'un partenariat politique avec la ville de Chengdu avant que la gestion n'en fut confiée à Éric Marié et qui connaissent un important succès vont dans ce sens (offre-formation.univ-montp1.fr/fr/_modules/education/education.html?educatio nid=FR_RNE_0341087X_PR_1247058274080). 